\title{
Modulating the endocannabinoid pathway as treatment for peripheral neuropathic pain: a selected review of preclinical studies
}

\author{
Shannon O'Hearn ${ }^{1}$, Patrick Diaz ${ }^{2}$, Bo Angela Wan ${ }^{2}$, Carlo DeAngelis ${ }^{2}$, Nicholas Lao ${ }^{1}$, Leila Malek ${ }^{2}$, \\ Edward Chow ${ }^{2}$, Alexia Blake ${ }^{1}$ \\ ${ }^{1}$ MedReleaf, Markham, Ontario, Canada; ${ }^{2}$ Odette Cancer Centre, Sunnybrook Health Sciences Centre, University of Toronto, Toronto, Ontario, \\ Canada \\ Contributions: (I) Conception and design: S O’Hearn, E Chow, A Blake; (II) Administrative support: BA Wan, L Malek, P Diaz; (III) Provision of \\ study materials or patients: N Lao; (IV) Collection and assembly of data: S O’Hearn, BA Wan, P Diaz, A Blake; (V) Data analysis and interpretation: \\ All authors; (VI) Manuscript writing: All authors; (VII) Final approval of manuscript: All authors. \\ Correspondence to: Ms. Shannon O’Hearn, MSc. MedReleaf Corp, Markham Industrial Park, Markham, Ontario, Canada. Email: sohearn@medreleaf.com.
}

\begin{abstract}
Chemotherapy-induced neuropathic pain is a distressing and commonly occurring side effect of many commonly used chemotherapeutic agents, which in some cases may prevent cancer patients from being able to complete their treatment. Cannabinoid based therapies have the potential to manage or even prevent pain associated with this syndrome. Pre-clinical animal studies that investigate the modulation of the endocannabinoid system (endogenous cannabinoid pathway) are being conducted to better understand the mechanisms behind this phenomenon. Five recent pre-clinical studies identified from Medline published between 2013 and 2016 were selected for review. All studies evaluated the effect of small-molecule agonists or antagonists on components of the endocannabinoid system in rats or mice, using cisplatin or paclitaxelinduced allodynia as a model of chemotherapy-induced neuropathic pain. Activation of the cannabinoid receptor-2 (CB-2) receptor by AM1710 blocked paclitaxel-induced mechanical and cold allodynia in one study. Four studies investigating the activation of both cannabinoid receptor-1 (CB-1) and CB-2 receptors by dual-agonists (WIN55,21 and CP55,940), or by the introduction of inhibitors of endocannabinoid metabolisers (URB597, URB937, JZL184, and SA-57) showed reduction of chemotherapy-induced allodynia. In addition, their results suggest that anti-allodynic effects may also be mediated by additional receptors, including TRPV1 and 5-hydroxytryptamine (5-HT $\left.\mathrm{H}_{\mathrm{A}}\right)$. Pre-clinical studies demonstrate that the activation of endocannabinoid CB-1 or CB-2 receptors produces physiological effects in animal models, namely the reduction of chemotherapy-induced allodynia. These studies also provide insight into the biological mechanism behind the therapeutic utility of cannabis compounds in managing chemotherapyinduced neuropathic pain, and provide a basis for the conduct of future clinical studies in patients of this population.
\end{abstract}

Keywords: Medical cannabis; cancer; pain; pre-clinical; chemotherapy; endocannabinoid; allodynia

Submitted Jul 04, 2017. Accepted for publication Aug 03, 2017.

doi: 10.21037/apm.2017.08.04

View this article at: http://dx.doi.org/10.21037/apm.2017.08.04

\section{Introduction}

Cannabis has been used for centuries to treat a variety of chronic conditions, including pain, nausea and vomiting, and spasticity $(1,2)$. Cannabis contains a variety of physiologically active compounds. Of these, tetrahydrocannabinol (THC) and cannabidiol (CBD) are two of the most well studied, both of which belong to a class of chemicals known as phytocannabinoids (3). THC and CBD produce different physiological effects through interactions with the body's endogenous signalling system known as the "endocannabinoid system" $(4,5)$. These compounds are becoming increasingly studied to determine their 
potential utility in the cancer setting for the management of neuropathy associated with chemotherapy (6). However, there is a limited amount of literature addressing the mechanism behind the physiological effects observed to be associated with cannabinoid administration. This select review aims to present the results of a number of pre-clinical studies that were conducted to investigate how modulation of the endocannabinoid system may affect chemotherapy-induced neuropathy in animal models.

\section{Materials and methods}

An informal literature search was conducted using search terms such as "endocannabinoid system" and "neuropathic pain". Five recently published (within the last 5 years) preclinical studies on the modulation of the endocannabinoid system in animal models were chosen for this selected review.

\section{Results}

\section{The endocannabinoid system}

The endocannabinoid system consists of a number of lipophilic ligands, primarily 2-arachidonoylglycerol (2-AG) and anandamide (AEA) (5). The two main target receptors of the endocannabinoid system include cannabinoid receptor-1 (CB-1) and cannabinoid receptor-2 (CB-2). Both receptors are found in the presynaptic membranes of neurons in the central and peripheral nervous systems, with CB-1 expressed in higher concentrations when compared to CB-2 in nervous tissue. Beyond the nervous system, CB-1 and $\mathrm{CB}-2$ have varying distributions in peripheral tissues and cell types (7). CB-1 can be found in numerous central and peripheral organs such as the spleen, lung, thymus, and heart, whereas CB-2 can be found more predominantly in cells of hematopoietic origin, including those of the immune system. More recently, cannabinoids have also been shown to bind and interact with additional receptor types, including the serotonin [5-hydroxytryptamine $\left.\left(5-\mathrm{HT}_{1 \mathrm{~A}}\right)\right]$ receptor, the vanilloid (TRPV1) receptors, and GPR55 receptors (8-11). 5-HT $\mathrm{T}_{1 \mathrm{~A}}$ receptors are part of the serotonin signalling pathway, which is involved in regulation of mood, appetite, and sleep (9). The TRPV1 receptor has been found in neurons involved in pain signalling, and the GPR55 receptor has been found in the dorsal root ganglion of the spinal cord, although its physiological role has not yet been identified (11).

Endogenous cannabinoid ligands produce a variety of effects through their interaction with target receptors. For example, 2-AG has been shown to activate CB-1, CB-2 and GPR55 receptors (5). AEA has approximately equal affinity for and activates CB-1, CB-2, and GPR55, in addition to TRPV1, but with a relatively lower affinity (12). Phytocannabinoids, THC and CBD also interact uniquely with the cannabinoid receptors. THC possesses similar properties to 2-AG, being an agonist of CB-1, CB-2, and GPR55. Therefore, it exerts physiological effects associated with activation of those receptor pathways, including neuroand immuno-modulation, which are likely to underlie its psychoactive and analgesic properties. On the other hand, CBD is an antagonist of CB-1, CB-2, and GPR55, but an agonist of TRPV1 and $5-\mathrm{HT}_{1 \mathrm{~A}}$ receptors $(2,12)$. In contrast to THC, CBD has been shown to produce antipsychotic, anxiolytic and anti-inflammatory effects (13).

The endogenous signalling molecules AEA and 2-AG are metabolized by the enzymes fatty acid amide hydrolase (FAAH) and monoacylglycerol lipase (MAGL), respectively (14). These two enzymes reduce endocannabinoid levels and therefore inhibit their signalling activity at CB-1 and CB-2 receptors. Interestingly, CBD acts as an inhibitor of FAAH (13). The complexity of interactions of cannabinoids and their receptors in vivo may lead to possible synergistic effects, which have been described as the "entourage effect" (15).

\section{Models of pain}

There are several different pain models that are routinely used to assess the effects of different therapies on peripheral neuropathic pain in pre-clinical studies. In the studies examined in this review, the most common pain models were cold and mechanical allodynia. These forms of allodynia were established by administering cisplatin or paclitaxel to test animals (rats or mice). Both of these substances are commonly administered chemotherapy drugs, known to generate peripheral nerve damage (16). The method used to assay the effect of a drug on the severity of allodynia varied between studies, but usually involved administering either mechanical or cold stimulation to the paws of test animals and comparing how often animals experiencing allodynia withdraw their paws, between treatment and control groups $(17,18)$. One study used a carrageenan model of inflammatory pain, in which oedema is induced by injecting carrageenan into the paws of test animals (19). Alleviation of this form of pain is determined by measuring paw thickness. This study also used a chronic constriction injury (CCI) 
Table 1 Summary of drugs that modulate the endocannabinoid system

\begin{tabular}{|c|c|c|}
\hline Receptor/€ & Agonists (reference) & Antagonists (reference) \\
\hline Receptors & ed with the endoca & nabinoid system \\
\hline CB-1 & $\begin{array}{l}\text { WIN55,21 (20); } \\
\text { CP55,940 (18) }\end{array}$ & AM251 (20) \\
\hline CB-2 & $\begin{array}{l}\text { AM1710 (18,20); } \\
\text { WIN55,21 (20); } \\
\text { CP55,940 (18) }\end{array}$ & AM630 $(20,21)$ \\
\hline 5-HT1A & - & WAY 100635 (9) \\
\hline Enzyme $\mathrm{m}$ & rs of endocannabin & ids 2-AG and AEA \\
\hline FAAH & - & $\begin{array}{l}\text { SA-57 (19); URB597 } \\
\text { (22); URB937 (22) }\end{array}$ \\
\hline MAGL & - & JZL184 (22); SA-57 (19) \\
\hline
\end{tabular}

CB-1, cannabinoid receptor 1; CB-2, cannabinoid receptor 2; 5-HT1A, serotonin receptor; FAAH, fatty acid amide hydrolase; MAGL, monoacylglycerol lipase; 2-AG, 2-arachidonoylglycerol; AEA, anandamide.

pain model, which involves the induction of mechanical allodynia by means of a surgical operation which constricts the sciatic nerve of the test animal. Alleviation of this form of pain was measured with a paw withdrawal test similar to those described above.

\section{Mechanisms of action}

The mechanisms of action of the drugs used in the studies examined in this review fall into two main categories, summarised in Table 1. The first category consists of drugs that act as direct agonists or antagonists to $\mathrm{CB}$ receptors. These can be CB-1 or CB-2-specific agonists [e.g., AM1710, a CB-2 agonist (18,21)], mixed CB-1/ CB-2 agonists [e.g., WIN55,21 (21)], or receptor-specific antagonists [e.g., AM251, a CB-1 antagonist]. The second category includes drugs that inhibit the breakdown of endogenous CB-1 and CB-2 agonists. For example, SA57 is a dual FAAH and MAGL inhibitor (20). Therefore, by inhibiting the breakdown of these compounds, SA-57 administration results in an increase in the amount of AEA and 2-AG available to bind CB-1 and CB-2 receptors. In addition to these mechanisms of action, some of the drugs examined in this review appear to have effects mediated by pathways other than those in the endocannabinoid system. For example, one study in mice found that CBD produced anti-allodynic effects that appeared to be predominantly mediated by its action at $5-\mathrm{HT}_{1 \mathrm{~A}}$ receptors (9).

\section{CB-1 mediated effects}

None of the drugs examined in this review had antiallodynic effects mediated solely via CB-1 receptors.

\section{$C B-2$ mediated effects}

Both Rahn et al. and Deng et al. studied the effect of AM1710, a selective CB-2-agonist, on paclitaxel-induced mechanical and cold allodynia $(18,21)$. Rahn et al. used a rat model while Deng et al. used a mouse model. In Rahn et al.'s study, administration of AM1710 at low $(0.032 \mathrm{mg} / \mathrm{kg} /$ day $)$ and high $(3.2 \mathrm{mg} / \mathrm{kg} /$ day $)$ doses reduced mechanical allodynia, while the high dose also reduced cold allodynia in paclitaxel-treated rats (21). The anti-allodynic effects were long-lasting, persisting for approximately 3 weeks after the cessation of AM1710 administration. When the CB-2 antagonist AM630 was co-administered with AM1710 the anti-allodynic effects of the drug disappeared, suggesting they are in fact mediated via CB-2 receptors. An analysis of cannabinoid receptor mRNA levels found that treatment with AM1710 increased the levels of CB-1 and CB-2 mRNA found in the rats, the effect of which was similarly blocked by administration of AM630, suggesting it is also medicated by CB-2 receptors. The study by Deng et al. made broadly similar discoveries in mice (18). AM1710 blocked mechanical and cold allodynia in wild type (WT) but not CB-2-knock out (KO) mice, or mice receiving the CB-2 antagonist AM630.

\section{CB-1/CB-2 mediated effects}

Guindon et al. performed a study of three compounds: the FAAH inhibitors URB597 and URB937, and the MGL inhibitor JZL184 (22). All three compounds were found to be capable of blocking cisplatin-induced mechanical and cold allodynia in rats. These effects were completely blocked by administration of either CB-1 or CB-2 antagonists, suggesting that both cannabinoid receptors were necessary, but not sufficient to produce anti-allodynic effects on their own. The effects of URB597 and URB937 also appeared to be mediated through vanilloid receptors, the mechanism of which is discussed below.

The mixed CB-1/CB-2-specific agonist WIN55,21 was also tested for its anti-allodynic properties by Rahn et al. (21). They found it was capable of blocking mechanical and cold allodynia induced by paclitaxel in rats. The coadministration of a CB-1 receptor antagonist (AM251) 
reversed the alleviation of mechanical allodynia, suggesting that the effect of WIN55,21 is predominantly mediated via CB-1 receptors. However, the cold allodynia effects could not be blocked by co-administration of CB-1 or CB-2 antagonists, suggesting the receptor pathway through which it is mediated is yet unknown.

Deng et al. performed a study of CP55,940, a mixed CB-1/CB-2 receptor agonist (18). They assessed the antiallodynic effects of the drug in wild-type as well as CB-1-KO and CB-2-KO mice. While WT and CB-2-KO mice displayed a similar reduction in allodynia in response to low-dose CP55,940 treatment, this response was not observed in $\mathrm{CB}-1-\mathrm{KO}$ mice, suggesting the anti-allodynic effect at low doses is mediated through CB-1 receptors. Assessment of the effect of high-doses of CP55,940 in WT mice was prevented by the fact that it induced catalepsy. However, in CB-1-KO mice a reduction in allodynia in response to high-dose CP55,940 was observed. This effect was blocked when CP55,940 was co-administered with a CB-2 antagonist, suggesting it is mediated by CB-2 receptors. CP55,940 showed similar affinities to CB-1 and CB-2 receptors in vitro suggesting that that the reason high-doses were required to see a CB-2-mediated effect on allodynia were due to the different concentrations of CB-1 and CB-2 receptors in the nervous system.

The dual FAAH/MGL inhibitor SA-57 was tested by Wilkerson et al. for its anti-oedema properties, as measured using the carrageenan inflammatory pain model, as well as for its anti-allodynic effects as measured using the CCI sciatic nerve model of neuropathic pain (19). Interestingly, all doses of SA-57 ranging from $1.25-12.5 \mathrm{mg} / \mathrm{kg}$ resulted in a maximal increase in AEA levels (from $4 \mathrm{pm} / \mathrm{g}$ in controls to $\sim 40 \mathrm{pm} / \mathrm{g}$ ) while 2 -AG levels ranged from $\sim 16 \mathrm{~nm} / \mathrm{g}$ in controls and at the lowest dose of SA-57, to $\sim 128 \mathrm{~nm} / \mathrm{g}$ at the highest administered dose of the drug. SA-57 was found capable of alleviating both oedema and mechanical allodynia in a dose dependant manner. The anti-allodynic effects were blocked in both CB-1-KO and CB-2-KO mice while the anti-oedema effects were blocked only in CB2-KO mice suggesting that the anti-allodynic effects are mediated by both receptors, while the anti-oedema effects are mediated primarily through CB-2 receptors.

\section{Other receptor effects}

As mentioned above, the FAAH inhibitors URB597 and URB937 were both found to have anti-allodynic effects that were blocked by antagonists of the vanilloid receptor
TRPV1 (22). This suggests that the anti-allodynic effects of cannabinoid agonists may at least partially be mediated via receptors other than CB-1 and CB-2. On this subject, Ward et al. found that CBD was capable of blocking paclitaxelinduced mechanical and cold allodynia in mice and that this effect was not blocked by CB-1 or CB-2 antagonists, but instead by co-administration of the $5-\mathrm{HT}_{1 \mathrm{~A}}$ receptor antagonist WAY 100635 (9).

\section{Discussion}

\section{Establishing mechanism of action}

Despite the lack of currently available options for managing chemotherapy-induced neuropathic pain, there are several drugs that appear promising as new treatments which have been identified in pre-clinical studies. However, it is necessary to understand the mechanism of action of these drugs before advancing to the conduct of large-scale clinical trials in patient populations.

The administration of AM1710 at multiple different doses demonstrated the ability to block both mechanical and cold allodynia through CB-2 receptor modulation, similar to the administration of CP55,940 which acts through CB-1 and CB-2 receptor activation (19,21). Additionally, Guindon et al. reported the ability of URB597 and URB937 to alleviate allodynia, which was mediated by CB-1, CB-2, and TRPV1 receptors in a mutually reinforcing manner, whereby blocking any one of the receptors using an antagonist completely mitigated the anti-allodynic effect of the drugs (22). These study findings suggest that targeted activation of receptors of the cannabinoid system has the potential to mitigate and even prevent neuropathy associated with chemotherapy, which should be further explored in clinical models.

Additionally, because the anti-allodynic effect of CBD appear to be mediated in part through $5-\mathrm{HT}_{1 \mathrm{~A}}$ receptors, it may also be possible to create drugs that strategically target these pathways as opposed, or in addition to those involving the cannabinoid receptors (9). This may result in the reduction of allodynia, while at the same time minimizing the psychoactive effects that may be induced by the activation of CB-1 or CB-2.

\section{Establishing negative side-effects}

Another important task will be to better understand 
the negative side-effects of drugs which modulate the cannabinoid pathways. In one study, researchers assessed whether cannabinoid receptor activation by AM1710 induced hypothermia, CB-1-mediated cannabinoid withdrawal, or motor dysfunction, none of which were observed (18). In another, CBD was found to produce no conditioned reward effect (9). SA-57 was even observed to generate heroin-sparing effects when it was used to treat mice with oedema and mechanical allodynia (19). However, some other studied treatments were associated with significant negative side-effects. For example, CP55,940 produces hypothermia and rimonabant-precipitated withdrawal effects in wild-type mice (17). Negative sideeffects were not measured in treatment groups receiving URB597, URB937, JZL189 and WIN55,212-2 in the remaining studies included in this review $(20,22)$.

\section{Conclusions}

Several pre-clinical studies have investigated the use of drugs that modulate pathways of the endocannabinoid and related systems. Such modulation may lead to treatment and even prophylaxis of peripheral neuropathic pain induced by common chemotherapeutic agents. In addition, insight into the mechanism of action of drugs targeted to these pathways will be invaluable for understanding how cannabinoid based therapies can be further developed and studied in clinical models. Additional research will be necessary to further establish the mechanism of action and potential negative side effects of these treatments, as well as to determine their clinical utility in this space where current treatment needs are not being met.

\section{Acknowledgements}

We thank the generous support of Bratty Family Fund, Michael and Karyn Goldstein Cancer Research Fund, Joey and Mary Furfari Cancer Research Fund, Pulenzas Cancer Research Fund, Joseph and Silvana Melara Cancer Research Fund, and Ofelia Cancer Research Fund. This study was conducted in collaboration with MedReleaf.

\section{Footnote}

Conflicts of Interest: The authors have no conflicts of interest to declare.

\section{References}

1. Whiting PF, Wolff RF, Deshpande S, et al. Cannabinoids for Medical Use: A Systematic Review and Meta-analysis. JAMA 2015;313:2456-73.

2. Grotenhermen F, Müller-Vahl K. The therapeutic potential of cannabis and cannabinoids. Dtsch Arztebl Int 2012;109:495-501.

3. Borgelt LM, Franson KL, Nussbaum AM, et al. The pharmacologic and clinical effects of medical cannabis. Pharmacotherapy 2013;33:195-209.

4. Pacher P, Bátkai S, Kunos G. The endocannabinoid system as an emerging target of pharmacotherapy. Pharmacol Rev 2006;58:389-462.

5. Mechoulam R, Parker LA. The endocannabinoid system and the brain. Annu Rev Psychol 2013;64:21-47.

6. Lynch ME, Cesar-Rittenberg P, Hohmann AG. A doubleblind, placebo-controlled, crossover pilot trial with extension using an oral mucosal cannabinoid extract for treatment of chemotherapy-induced neuropathic pain. J Pain Symptom Manage 2014;47:166-73.

7. Luongo L, Maione S, Di Marzo V. Endocannabinoids and neuropathic pain: focus on neuron-glia and endocannabinoid-neurotrophin interactions. Eur J Neurosci 2014;39:401-8.

8. Ross RA. Anandamide and vanilloid TRPV1 receptors. $\mathrm{Br}$ J Pharmacol 2003;140:790-801.

9. Ward SJ, McAllister SD, Kawamura R, et al. Cannabidiol inhibits paclitaxel-induced neuropathic pain through 5-HT(1A) receptors without diminishing nervous system function or chemotherapy efficacy. Br J Pharmacol 2014;171:636-45.

10. Ryberg E, Larsson N, Sjögren S, et al. The orphan receptor GPR55 is a novel cannabinoid receptor. $\mathrm{Br} \mathrm{J}$ Pharmacol 2007;152:1092-101.

11. Lauckner JE, Jensen JB, Chen HY, et al. GPR55 is a cannabinoid receptor that increases intracellular calcium and inhibits M current. Proc Natl Acad Sci U S A 2008;105:2699-704.

12. Lu Y, Anderson HD. Cannabinoid signaling in health and disease. Can J Physiol Pharmacol 2017;95:311-27.

13. Zhornitsky S, Potvin S. Cannabidiol in humans-the quest for therapeutic targets. Pharmaceuticals (Basel) 2012;5:529-52.

14. Huang WJ, Chen WW, Zhang X. Endocannabinoid system: Role in depression, reward and pain control (Review). Mol Med Rep 2016;14:2899-903. 
15. Russo EB. Taming THC: potential cannabis synergy and phytocannabinoid-terpenoid entourage effects. Br J Pharmacol 2011;163:1344-64.

16. Windebank AJ, Grisold W. Chemotherapy-induced neuropathy. J Peripher Nerv Syst 2008;13:27-46.

17. Deng L, Cornett BL, Mackie K, et al. CB1 Knockout Mice Unveil Sustained CB2-Mediated Antiallodynic Effects of the Mixed CB1/CB2 Agonist CP55,940 in a Mouse Model of Paclitaxel-Induced Neuropathic Pain. Mol Pharmacol 2015;88:64-74.

18. Deng L, Guindon J, Cornett BL, et al. Chronic cannabinoid receptor 2 activation reverses paclitaxel neuropathy without tolerance or cannabinoid receptor 1-dependent withdrawal. Biol Psychiatry 2015;77:475-87.

19. Wilkerson JL, Ghosh S, Mustafa M, et al. The endocannabinoid hydrolysis inhibitor SA-57: Intrinsic antinociceptive effects, augmented morphine-induced antinociception, and attenuated heroin seeking behavior in

Cite this article as: O'Hearn S, Diaz P, Wan BA, DeAngelis C, Lao N, Malek L, Chow E, Blake A. Modulating the endocannabinoid pathway as treatment for peripheral neuropathic pain: a selected review of preclinical studies. Ann Palliat Med 2017;6(Suppl 2):S209-S214. doi: 10.21037/ apm.2017.08.04 mice. Neuropharmacology 2017;114:156-67.

20. Niphakis MJ, Cognetta AB 3rd, Chang JW, et al. Evaluation of NHS carbamates as a potent and selective class of endocannabinoid hydrolase inhibitors. ACS Chem Neurosci 2013;4:1322-32.

21. Rahn EJ, Deng L, Thakur GA, et al. Prophylactic cannabinoid administration blocks the development of paclitaxel-induced neuropathic nociception during analgesic treatment and following cessation of drug delivery. Mol Pain 2014;10:27.

22. Guindon J, Lai Y, Takacs SM, et al. Alterations in endocannabinoid tone following chemotherapy-induced peripheral neuropathy: effects of endocannabinoid deactivation inhibitors targeting fatty-acid amide hydrolase and monoacylglycerol lipase in comparison to reference analgesics following cisplatin treatment. Pharmacol Res 2013;67:94-109. 